\title{
Innovations
}

\section{Using the CTC tool in establishing mid-levels of expanded outcomes framework - A CME provider perspective}

\section{Authors}

\section{Farhan Vakani'; W. Daniel Cogan'}

\section{Abstract}

Existing gaps in the quality of healthcare have led to calls for change by Continuing Medical Education (CME) providers around the world to plan and implement continuing medical education activities based on improving physician competence and performance. This article offers the use of the commitment to practice change (CTC) tool at mid-levels of the expanded outcomes framework using post-only design, for inquiring and promoting physicians' commitment to practice change, and for assessing the impact of the educational activity.

\section{Keywords}

commitment to change; expanded outcomes cramework; physicians

\section{Expanded outcomes framework}

In the past few years there has been a marked shift toward focusing continuing medical education (CME) activities on improving competence and performance, rather than on simply providing information or opportunities for face-to-face discussions. Moore's expanded outcomes framework is an example of an idealized model for planning and assessing CME with a specific focus for improving physicians' competence, practice, and healthcare outcomes. This model is designed to work in an expanded fashion, to include practice and real world applications, such as physician competence and performance (levels 4 \& 5), and patient and population health (levels $6 \& 7$ ), after attaining the lower levels of the framework (level $3=k$ knowledge, level $2=$ satisfaction, and level $1=$ attendance).

However, the accreditation agencies for healthcare providers are becoming more appreciative of and are more demanding of the use of the higher levels of expanded outcomes framework in CME evaluation, rather than the lower levels, which are becoming less important in today's innovative and technological world of CME.'

\section{Commitment to practice change (CTC) tool}

The commitment to practice change (CTC) tool is used by CME providers to promote inquiry and commitment to change among physicians and to more effectively assess the impact of education on

\footnotetext{
' University of Louisville School of Medicine, Louisville, Kentucky, USA

*E-mail: vakani@gmail.com
}

\begin{abstract}
Cite this article as:
Vakani F, Cogan WD. Using the CTC tool in establishing midlevels of expanded framework - A CME provider perspective. Innovations in Global Health Professions Education. 2015:7. http://dx.doi.org/10.20421/ighpe2015.7

This is an open access article distributed under the terms of the Creative Commons Attribution license CC BY 4.0, which permits unrestricted use, distribution and reproduction in any medium, provided the original work is properly cited.
\end{abstract}


their clinical practice. ${ }^{2}$ The distinguishing feature of the CTC approach is that it asks for and documents participant physician statements of intention about practice change and performance relative to what was taught in the CME activity. The CTC tool is mainly used to gather information or statement of intent results just after the activity (known as post-only design). The next phase, a post/follow-up design, is many times more difficult to implement. The participants are queried much later in their practice settings to confirm their compliance and progress in applying previously identified behavioral changes, as well as reasons for non-compliance. The identification of non-compliance factors or barriers may advance understanding for future, improved educational interventions. Usually the statements of intention or the results obtained just after the event can be helpful for the CME providers to compare and analyze the objectives of the course, content of the activity, instructional strategy, examples or case studies incorporated into the content, and the allocation of time to each content area. $^{3}$

In using the CTC tool in establishing mid-levels of expanded outcomes, CME providers understand and recognize that the translation of new knowledge into clinical practice can be a slow and arduous process. Existing gaps in the quality of healthcare have led to calls for change by providers around the world to plan and implement CME activities based on improving physician competence and performance, rather than based on the traditional transfer of knowledge, whether declarative or procedural. This shifting environment has caused us to think about a valid, reliable, and feasible planning tool that can be combined with an effective evaluation plan that can be carried out at the end of the event or implemented as a separate component, for asking commitment or plans to practice change, and the impact of the educational activity on physicians.

The distinguishing feature of the widely accepted CTC tool is that it 1) promotes a sense of responsibility and ownership among physician respondents with regard to their post-CME activity performance; 2) enhances their qualities of courage and resolve by giving them the confidence to do honest self-assessment; 3 ) makes them responsible for the changes they propose to make as a result of what they learned during the activity; and 4) somehow also assesses the level 5 (i.e. performance), as a post-follow-up design to assess compliance with the pre-statements or commitments that otherwise have been assessed through performance improvement initiatives.

While there are significant gaps in the literature conceptualizing the CTC approach, the positive attributes of this tool take precedence over its limitations. The purpose of this perspective is to encourage CME providers to consider using the CTC tool at mid-levels of the expanded outcomes framework, ${ }^{3}$ thus, drawing attention to the need to promote performance and/or competence (levels $5 \& 4$ ), by encouraging participants to commit to implementing those self- identified changes in their practices.

\section{References}

1. Moore DE, Green JS, Gallis HA. Achieving desired results and improved outcomes: integrating planning and assessment throughout learning activities. Journal of Continuing Education in the Health Professions. 2009; 29(1):1-15.

2. Evans JA, Mazmanian PE, Dow AW, Lockeman KS, Yanchick VA. Commitment to Change and Assessment of Confidence: Tools to Inform the Design and Evaluation of Interprofessional Education. Journal of Continuing Education in the Health Professions. 2014; 34(3):155-163.

3. Shershneva MB, Wang M-F, Lindeman GC, Savoy JN, Olson CA. Commitment to practice change: an evaluator's perspective. Evaluation $\&$ the health professions. 2010; 33(3):256-275. 